\title{
Controlled Water Intake: A Method for Objectively Evaluating Thirst and Hydration State in Monkeys by the Measurement of Blood Osmolality
}

\author{
Hiroshi Yamada, Kenway Louie, and Paul W. Glimcher \\ Center for Neural Science, New York University, New York, NY 10003, USA.
}

\section{Abstract}

Standard methods for behavioral and neurophysiological experiments in the non-human primate rely on controlled water access as a means for motivating subject performance. It is, however, still not clear whether animals are able to regulate their fluid balance appropriately under these experimental settings. Further, the physical state associated with a subject monkey's thirst has not yet been objectively assessed under these conditions. Both of these deficiencies arise from the lack of a method for independently evaluating the hydration state of these subjects during experimental testing. To address these limitations, we measured the blood osmolality, the most widely used hematological index of hydration status, of three rhesus monkeys under conditions of controlled water access while they participated in a standard reinforced behavioral task for fluid rewards. We found that day-to-day hydration levels, as measured by serum osmolality, appears to be well regulated in a narrow range of values ( 300 to $320 \mathrm{mOsmo} / \mathrm{KgH} 2 \mathrm{O}$ ) by experimental subjects under these conditions: Animals work harder and longer to earn more water rewards on a day when they are in a lower hydration state (higher osmolality) than when they are in a higher hydration state (lower osmolality). We also found that osmolality level decreases almost immediately after water intake, within $30 \mathrm{~min}$, in a surprisingly linear manner. Osmolality thus seems to provide a fairly precise reflection of the monkeys' hydration state on a timescale of minutes. This evidence suggests that osmolality can be used as a tool for monitoring the hydration level of experimental subjects.

\section{Keywords}

Non-human primate; Thirst; hydration state; Blood osmolality

\section{Introduction}

Over the course of the last several decades monkeys have been used as subjects in a wide range of behavioral and neurophysiological experiments. In a typical experiment, animals are trained to make some movement or assess some stimulus in return for a fluid reward (Evarts, 1968; Goldberg and Wurtz, 1972). In order to maximize the reinforcement provided

\footnotetext{
C 2010 Elsevier B.V. All rights reserved.

Corresponding Author: Hiroshi Yamada, Center for Neural Science, New York University, 4 Washington Place, Room809, New York, NY, 10003, Tel: (212) 998-3904, Fax: (212) 995-4011, hyamada@nyu.edu.

Publisher's Disclaimer: This is a PDF file of an unedited manuscript that has been accepted for publication. As a service to our customers we are providing this early version of the manuscript. The manuscript will undergo copyediting, typesetting, and review of the resulting proof before it is published in its final citable form. Please note that during the production process errors may be discovered which could affect the content, and all legal disclaimers that apply to the journal pertain.
} 
by fluid rewards (and hence the animal's motivation to perform the task), these monkeys are typically allowed only limited access to fluids when they are not in the experimental setting.

While this approach has been highly successful, in light of the many measurements of behavioral and neuronal data it has yielded, it also poses several questions that remain unanswered. First, an implicit assumption that is often (although not always) made for the purpose of data analysis is that the subjective values of fluid rewards change only very slightly throughout the data collection period. The effect of changes in hydration state is implicitly neglected or assumed to vary randomly with regard to all variables of interest over a period of neuronal measurement despite the fact that the values of primary rewards change depending on the physical state of a subject (Lau and Glimcher, 2008; Platt and Glimcher, 1999; Samejima et al., 2005). A second question posed by this method regards the physiological state of the animals at the time of testing. Are the procedures to control water intake appropriate for maintaining the health of the subjects? Are animals able to regulate their fluid balance appropriately under these conditions, or does restricted water access compromise the ability of the animals to maintain their health? A final question that arises is whether the subjective values of fluid rewards, as modulated by the actual thirst of the animals, can be directly measured and manipulated in an effort to better understand the neural basis of decision-making.

A growing body of evidence indicates the scientific importance of this last question. The activity of brain regions representing the subjective values of rewards is known to depend on whether subjects are sated or not, and the neural representation of foods and fluids has been shown to change after ingestion in both humans and animals (Critchley and Rolls, 1996; de Araujo et al., 2003; Simon et al., 2006). In related work, the motivation level associated with rewards, as measured from the behavioral responses of rats and monkeys, has been observed to decrease after rewards are consumed (Dickinson and Balleine, 1994; Minamimoto et al., 2009). Indeed, even the degree of risk preference evidenced by at least one species of bird has been argued to be strongly affected by hunger level (Caraco et al., 1980).

To address all of these issues, we directly assessed the blood osmolality of non-human primates involved in a decision-making task over an extended period, and related these measurements to behavior and body weight. Osmolality is a measure of the number of osmoles of solute per kilogram of solvent (Stedman, 2006). Abundant physiological evidence suggests that under many (but by no means all) conditions thirst and the desire for fluids is proportional to blood osmolality (Bernardis and Bellinger, 1996; Berne and Levy, 1993; Rolls and Rolls, 1982). In addition to its relevance to thirst and metabolic state in the nonhuman primate (Wood et al., 1982), it should be noted that osmolality is easily and precisely measured in the laboratory (Armstrong, 2005) using a freezing-point method that can be conducted on samples of blood plasma as small as $20 \mathrm{ul}$ in volume (eg.: www.aicompanies.com). For these reasons, regular measurements of osmolality should, at least in principle, allow us to address the three questions posed above. Osmolality measurements should allow us to i) determine how quickly and how much the hydration state of monkey subject changes as a function of fluid consumption over a typical period of data collection within a day, ii) determine the fluctuation of hydration state of the animals across multiple days under a controlled water access protocol, and iii) determine whether osmolality can be used as an objective measure of thirst and, accordingly, of the subjective value of fluid rewards in the laboratory.

Our results suggest that osmolality can, in fact, be used as a tool for monitoring the hydration level of experimental subjects. Subjects in this protocol do appear to regulate blood osmolality behaviorally within a fixed narrow range suggesting that animals participating in these experiments select and maintain target osmolalities. Our data also 
suggest that blood osmolality may serve as an easily quantified physiological measure of the physical state of thirst for future studies of decision-making and reward.

\section{Materials and Methods}

\section{Subjects and Surgical Procedures}

Three rhesus monkeys were used (Macaque mulatta; Monkey DE, $7.2 \mathrm{~kg}$, 5 years Monkey $\mathrm{HU}, 8.0 \mathrm{~kg}, 5$ years, Monkey BT, $6.5 \mathrm{~kg}, 5$ years). All experimental procedures were approved by the New York University Institutional Animal Care and Use Committee and performed in compliance with the Public Health Service's Guide for the Care and Use of Animals. Prior to training, each animal was implanted with a head-restraint prosthesis (Platt and Glimcher, 1997) and a scleral eye coil (Fuchs and Robinson, 1966) (except monkey BT who did not receive an eye coil). Surgical procedures were performed using aseptic techniques under general anesthesia (Platt and Glimcher, 1997).

\section{Water access control}

In the approved controlled water access protocol we employed, monkeys received at least a fixed minimal daily allocation of the water determined independently for each individual as below. This minimum daily allocation was required to be delivered in at least two separate daily rations. The smaller of these two rations was required to be no less than $25 \%$ of the fixed minimal daily allocation of water. (If a monkey, as an example, obtained $85 \%$ of his minimal daily allocation during the behavioral task, he would consume $110 \%(85 \%+25 \%)$ of his minimal daily allocation in total that day.) The two rations were required to be timed such that no animal was without water for more than 16 hours. Finally, all animals were required to receive a 'large' amount of water at least once a week (where 'large' is defined as more than two to three times the minimal daily allocation). The monkeys typically received 700 to $1000 \mathrm{ml}$ water each Friday evening. It was under these conditions that we assessed blood osmolality.

The minimal daily allocation for behaviorally trained animals performing tasks for fluid reward was determined for each individual monkey in an Animal Welfare Committee (AWC)-approved process: On weekdays, monkeys obtained water in the experimental test room while engaged in a reinforced behavioral task for as long as they desired. Once stable behavioral performance had been established under these conditions, measurements of water intake during the behavioral task were made on two consecutive days. The minimal daily allocation for each monkey was then defined as the combined total amount of the water earned by engaging the experiment in over two consecutive days (we typically employ a Monday and a Tuesday). The intent of this procedure was to insure that animals received no less than twice the daily fluid for which they are willing to work. It should be noted that this procedure for assessing the minimum daily allocation could be repeated, a repetition which allowed us to set new minimums as the animals grew. Measured in this way, the daily minimal water allocation for the two principal animals during the period that this study was underway was fixed at $120 \mathrm{ml}$ in monkey DE $(7.2 \mathrm{~kg})$ and $140 \mathrm{ml}$ in monkey $\mathrm{HU}(8.0 \mathrm{~kg})$.

To broaden our sampling procedure, the AWC allowed us to examine a third monkey who had not been behaviorally trained in this way. Instead of engaging in a reinforced behavioral task, monkey BT obtained water from a sipper-tube while seated in a primate chair in the experimental room. Water was automatically supplied to the animal at $1 \mathrm{~s}$ to 2 min intervals. If the monkey stopped drinking for approximately $10 \mathrm{~min}$, water intake for that day was considered completed and that volume (measured on two successive days) was used, as described above, to set the daily minimum allocation for that animal. This monkey, subject BT, received a daily minimal water allocation of $120 \mathrm{ml}(6.5 \mathrm{~kg})$. 
The average amount of water consumed daily by each monkey during the period of this study (distributed over the two separate daily rations) were: Monkey DE; $140 \mathrm{ml} / \mathrm{day}$ or 19.4 $\mathrm{ml} / \mathrm{kg} /$ day, Monkey HU; $180 \mathrm{ml} /$ day or $22.5 \mathrm{ml} / \mathrm{kg} /$ day, Monkey BT; $120 \mathrm{ml} /$ day or $18.5 \mathrm{ml} /$ day/kg.

\section{Behavioral Testing: Eye Movement Task}

All behavioral tests were conducted in a dimly lit sound-attenuated room. Eye movements were measured using a scleral coil (Fuchs and Robinson, 1966) and sampled at $500 \mathrm{~Hz}$. Visual stimuli were generated using light-emitting diodes (LEDs) $110 \mathrm{~cm}$ from the monkeys' eyes. Monkeys were seated in a standard primate chair and performed a standard delayed saccade task (Mays and Sparks, 1980). Each trial started with a $300 \mathrm{~ms} 500 \mathrm{~Hz}$ tone, after which the monkey was given $1000 \mathrm{~ms}$ to align its gaze within 3 degree of a red LED in the center of the visual field. After maintaining fixation for 200 to $500 \mathrm{~ms}$, one peripheral red LED was illuminated at either 8 or 12 degrees in one of 8 possible directions $(0,45,90$, $135,180,225,270,315$ angular degrees). $200 \mathrm{~ms}$ to $800 \mathrm{~ms}$ later, the central fixation point disappeared, instructing the monkeys to make a saccade to the peripheral red LED by shifting gaze to within 4 degrees of its location. Reward water (about $0.15 \mathrm{ml}$ ) was delivered by solenoid valve $300 \mathrm{~ms}$ after the eye movement was completed. Reward delivery was followed by a $600 \mathrm{~ms}$ to $1500 \mathrm{~ms}$ inter-trial interval.

\section{Blood Collection}

The monkeys were seated in a standard primate chair and desensitized to leg restraint by the use of positive reinforcement with food rewards before starting blood collection. Blood samples of $1.5 \mathrm{ml}$ were drawn from the saphenous vein via a butterfly needle (experiment 1) or a venous catheter placed for the duration of a single day's sampling (experiment 2). All animals were sampled while awake and (after desensitization) showed no distress during the sampling procedure. At least $0.5 \mathrm{ml}$ serum was extracted from the $1.5 \mathrm{ml}$ blood sample by centrifugation at $3400 \mathrm{rpm}$ for $5 \mathrm{~min}$. The extracted serum was sent by refrigerated transportation to a commercial laboratory for osmolality determination (Cornell University Clinical Pathology Laboratory). The total amount of blood extracted within any two week experimental period did not exceed $5 \%$ of total blood volume (total blood volume estimated as $65 \mathrm{ml} / \mathrm{kg}$ weight).

\section{Weight measurements}

From Monday to Friday, the body weight of each monkey was measured before blood collection and following performance of the eye movement task.

\section{Experiment 1: Measurement of blood osmolality across a week-Blood} osmolality from Monday to Friday was measured before the animals performed the behavioral task for fluid rewards on each day. To accomplish this, a single sample of blood was collected at the same time each day ( \pm 60 minutes), either in the morning (Monkey DE and Monkey HU) or in the afternoon (Monkey BT). About thirty minutes after blood collection, monkeys DE and HU began to perform the eye movement task for water rewards. Monkey BT obtained his minimal daily allocation $(120 \mathrm{ml})$ in his cage: $90 \mathrm{ml}$ water after he went back to its cage in the afternoon and $30 \mathrm{ml}$ water in the morning about 5 hours before the testing. In each of the three monkeys, these 5 measurements made over the course of a week were repeated twice on non-consecutive weeks.

\section{Experiment 2: Measurement of blood osmolality after water intake within a}

day-On a Friday when the monkeys were not engaged in experiment 1, we performed four blood collections over the course of $1.5 \mathrm{~h}$ while animals gradually received water. In this 
experiment, samples were drawn using standard vacuum blood collection tubes from a single intravenous catheter inserted into a saphenous vein at the start of the session and removed before the monkey was returned to its cage at the end of the session. Blood samples were gathered at $30 \mathrm{~min}$ intervals by inserting a long needle into the catheter that projected just outside the tip of the catheter into the vein itself. After a sample was gathered the animal consumed $50 \mathrm{ml}$ of water from a bottle over one minute. The next blood sample was then gathered $30 \mathrm{~min}$ after the preceding sample. This sequence was repeated until 4 samples had been gathered. This process roughly mimicked the gradual hydration that occurs during a standard reinforced behavioral task and allowed us to assess the effect of such gradual hydration on blood osmolality.

\section{Measurement of baseline value of blood osmolality in each individual}

The blood osmolality of our subjects when access to water was not controlled was also measured to provide a baseline value for each of the subjects studied here. To derive this measure, each of the monkeys was offered free water access. One liter of water every morning and afternoon was delivered in their home cages for a period of four weeks. (Thus animals could choose to consume a maximum of two liters of water on each day of the fourweek period). Three blood samples were then collected from each monkey under the free water access condition in the mornings of three separate days. Note that these measurements were made after the conclusion of experiments 1 and 2 .

\section{Statistical Analysis}

Changes in serum osmolality across a week (experiment 1 ) and within a day (experiment 2) were analyzed by two-way ANOVA at $P<0.05$ (experiment 1: monkey and day of week, experiment 2: monkey and water intake). The changes in osmolality from Monday baseline were compared to the other days of week by two sample t-test at $P<0.05$. The changes in osmolality from the first sample in a day were analyzed using linear regression.

In experiment 1 , the influence of serum osmolality on water intake during the eye movement task was analyzed by a general linear model. The water Intake $(Y)$ was fitted using the following estimator:

$$
Y=b_{0}+b_{1} \mathrm{SO}+b_{2} \text { Monkey }+b_{3} \text { Interaction }+ \text { error }
$$

where $b_{0}$ and error are the intercept and residual, respectively. SO is the serum osmolality. "Monkey" is a categorical variable identifying monkeys DE and HU. "Interaction" is the interaction between SO and "Monkey". If the $b_{1}$ or $b_{3}$ is not 0 at $P<0.05$, then we can conclude that the water intake was significantly affected by the serum osmolality.

The influence of serum osmolality on weight change from Monday was analyzed by a second general linear model. The weight change from Monday $(Y)$ was fitted using following estimator:

$$
Y=b_{0}+b_{1} \mathrm{SO}+b_{2} \text { Monkey }+b_{3} \text { Interaction }+ \text { error }
$$

where $b_{0}$ and error are the intercept and residual, respectively. SO is the serum osmolality. "Monkey" is again a categorical variable, this time identifying monkeys DE, HU, and BT. "Interaction" is the interaction between SO and Monkey. If the $b_{1}$ or $b_{3}$ is not 0 at $P<0.05$, then we can conclude that the weight change from Monday was significantly affected by the serum osmolality. 


\section{Results}

\section{Experiment 1: Blood Osmolality Across a Week}

We measured day-to-day changes in blood osmolality over the course of a week as monkeys participated in a standard reinforced behavioral task under conditions of controlled water access. Measurements were made during two non-consecutive weeks for each of three monkeys. We found that hydration states, as indicated by serum osmolality, were weakly idiosyncratic under these conditions; osmolality levels were slightly but significantly different from monkey to monkey (Fig. 1A, two-way ANOVA, P = 0.027). Figure 1 presents the serum osmolality measurements for all three animals (monkey DE-circles, monkey HUdiamonds, and monkey BT-squares). Irrespective of the variance in absolute measurements of osmolality across individuals, however, serum osmolality showed a significant relative increase from Monday to Tuesday (Fig 1B) but then settled to a roughly stable average level for the remaining three weekdays (Fig. $1 \mathrm{C}$, two-way ANOVA, $\mathrm{P}=0.249$, interaction: $\mathrm{P}=$ 0.334). While the osmolality did not seem to increase from Monday to Tuesday in some cases (see white circles and white squares), the average increase from Monday to Tuesday may reflect an underlying gradual increase in osmolality resulting from the controlled water access protocol, which was implemented after the monkeys were fully hydrated the previous Friday.

Do changes in serum osmolality precisely reflect changes in the hydration state of each monkey and do monkeys regulate their osmolality by deciding how much water to earn during a standard behavioral task? In monkey DE (see, Fig.1. white circles), for example, serum osmolality remained low $(\sim 300 \mathrm{mOsmo} / \mathrm{KgH} 2 \mathrm{O})$ on Monday and Tuesday. Then, osmolality levels rose to relatively fixed higher level $(\sim 305 \mathrm{mOsmo} / \mathrm{KgH} 2 \mathrm{O})$ on subsequent days of the week. Interestingly, changes in the monkeys' behavior paralleled these changes in osmolality. On the two days in which osmolality was at its minimum level, the monkey chose to drink almost nothing (only about 30 trials on Tuesday over a period of less than 20 $\mathrm{min}$ ), refusing to engage in the behavioral task (Fig. 2A, two white circles at bottom left). In contrast, water intake during the behavioral task was higher on the three subsequent days in which osmolality was relatively stable at a higher level. For example, he obtained $110 \mathrm{ml}$ of fluid reward on Thursday by successfully completing 706 trials over about one and one-half hours. In general we thus observed that the average water intake during the behavioral task increased sharply from Monday to Tuesday (Fig. 1D) and appeared to occur in parallel with the increase of serum osmolality (Fig. 1C). The supplemental water monkeys received out of the task, of course, also decreased throughout the week as specified in the methods section.

To quantitatively examine whether there was a significant relationship between blood osmolality and the water consumption behavior of the monkeys during the task, we used a general linear model analysis. The effect of serum osmolality measured before the task, individual differences between monkeys, and the interaction between these variables were assessed. We found that the serum osmolality measured before the task significantly influenced the water intake. (Serum Osmolality: $P<0.001$, Monkey: $P=0.682$, interaction: $P=0.974)$. Indeed, we found a strong and significant positive correlation between osmolality and water consumed in the behavioral task for each monkey (Fig. 2A, Monkey DE: $\mathrm{r}=0.745, P=0.013$, Monkey HU: $\mathrm{r}=0.721, P=0.019)$. Thus, the monkeys appeared to regulate their hydration state by deciding how long they wished to engage in the behavioral task.

We also found that day-to-day fluctuations in blood osmolality also affected the fluctuations in weight (weight change from Monday baseline) assessed across our small population of subjects as is often hypothesized (Fig. 2B, Serum Osmolality: $P<0.001$ ). Note however that the decrement of weight in monkey DE was smaller than the others (Monkey: $P=0.024$ ). 
This suggest that the effect of osmolality on weight-change might be slightly idiosyncratic to each individual, although there is no statistical significance to this idiosyncrasy (interaction: $P=0.228)$. The correlations between the weight changed and serum osmolality were: Monkey DE: $\mathrm{r}=-0.237, P=0.510$ : Monkey HU: $\mathrm{r}=-0.777, P=0.008$ : Monkey BT: $\mathrm{r}=$ $-0.586, P=0.0751$. To summarize, these data suggest that daily measurements of animal weight can serve as a proxy for osmolality when one wishes to track hydration state, although the power of that measurement may vary to some extent from individual to individual.

A final question we wished to address with this experiment was whether the method we used to define the minimum amount of water required by each animal each day permitted stable regulation of blood osmolality. Monkey BT, for this purpose, received the minimal daily allocation over the course of two non-consecutive weeks without participating in a behavioral task. The data in figure 1A (squares) indicate that his osmolality level remained within a physiological range over the course of the entire week. The osmolality never showed a continuous rise over the course of the week. These data thus suggest that our definition of the minimal daily allocation (roughly $20 \mathrm{ml} / \mathrm{kg}$ daily) provides sufficient fluid to stably maintain the osmotic state of our subjects' blood serum.

Although the data presented above indicate that under our protocol the monkeys could each effectively maintain their hydration state within a fixed range, these data cannot tell us what hydration state was maintained by these same subjects off-study. To determine what blood osmolality these subject maintained when they had free access to water, we measured blood osmolality under free access conditions as a baseline value. The average baseline value for serum osmolality was $8 \mathrm{mOsmo} / \mathrm{KgH} 2 \mathrm{O}$ lower (from 310 to $302 \mathrm{mOsmo}$ ) than under the controlled water access procedure described above (Fig.3, two-way ANOVA, P < 0.001, Monkey: $\mathrm{P}=0.380$, interaction: $\mathrm{P}=0.330$ ). The controlled water access thus caused roughly $2.5 \%$ increase in serum osmolality from the off-study level. Although there was no significant difference of the effect among monkeys, controlled water access seemed to have its largest effect in monkey BT. Note however, that there was also a high degree of variation in off-study osmolality for some individuals as indicated in Figure 3.

\section{Experiment 2: Blood Osmolality After Water Intake, Within a Day}

The results from experiment 1 suggest that daily measurements of blood osmolality can predict how much monkeys will choose to work to obtain water rewards over the course of that day. But how quickly do blood osmolality levels respond to water intake and can minute-by-minute measurements be used to perform a finer analysis of hydration state and its relation to behavior? To begin to examine this issue, we measured serum osmolality after a series of small water intakes that roughly mimicked the schedule of water intake monkeys xperience during a typical behavioral experiment. We gathered 4 blood samples at 30 minute intervals, with each of the first 3 of these samples followed by the oral delivery of 50 $\mathrm{ml}$ of water. We again found that individual monkeys showed idiosyncratic absolute osmolality levels as in experiment 1 (Fig. 4A, two-way ANOVA, P < 0.001). For all animals, however, osmolality level decreased as a function of water intake in a surprisingly linear manner over the course of the experimental session (Fig. 4B, linear regression, $\mathrm{P}<$ 0.001), while the absolute osmolality levels of the animals also decreased in response to each $30 \mathrm{~min}-50 \mathrm{ml}$ administration of water (Fig. 4C, two-way ANOVA, P = 0.004, interaction: $\mathrm{P}=0.334$ ). On average, serum osmolality decreased about $8 \mathrm{mOsm} / \mathrm{KgH} 2 \mathrm{O}$ over the course of a $150 \mathrm{ml}$ delivery that was provided over the $90 \mathrm{~min}$ experimental period. (Average monkey weight: $7.23 \mathrm{~kg}$.) This result suggests that blood osmolality decreases as a rapid function of water intake with a time-course relevant to the duration of a typical behavioral task. This thus suggests that during a standard behavioral task that lasts for a few 
hours, fluid satiety likely determines when monkeys choose to stop engaging in the available reinforced task.

\section{Discussion}

We found, for the first time, evidence that the fluid balance of monkeys under controlled water access is reflected in their blood osmolality level. Day-to-day fluctuations in hydration state, as indicated by osmolality level, can predict how much water monkeys will choose to consume during a standard behavioral task (Fig. 2A). Animals work harder and longer to earn more water on a day when they are in a lower hydration state (higher osmolality) than when in a higher hydration state (lower osmolality).

This relationship between osmolality level and water consumption behavior doubtless reflects the physiology of fluid balance. The osmoreceptors located around the anteriorventral third ventricular (AV3V) region of the hypothalamus are known to directly detect changes in blood osmolality (Buggy et al., 1979). Neurons of the organum vasculosum of the lamina terminalis (OVLT), for example, directly respond to increases and decreases in blood osmolality (Thrasher and Keil, 1987) and these changes are known to stimulate or suppress the ingestion of both water and salt. These physiological mechanisms indicate that blood osmolality is one of the physiological variables that directly controls the behavioral regulation of hydration state in monkeys. Another important mechanism is the reninangiotensin system. It is known that reduction of blood volume stimulates fluid ingestion behavior and the suppression of diuresis through this well described pathway (Berne and Levy, 1993). Osmolality is thus a critical component of hydration state and the direct measurement of blood osmolality therefore provides one way, but by no means the only way, to quantify hydration state. Given the current technological capability to quickly measure osmolality from small blood samples, measurements of osmolality provide an excellent technique for monitoring fluid balance in awake-behaving animals.

In our animals, we observed that the hydration level went up as a step function from Monday to Tuesday, and then settled to a stable level. While there were differences in absolute osmolality level from week-to-week and from animal-to-animal, we observed that osmolality remained within a limited range associated with what appears to be a stable hydration state in each of the monkeys despite the challenge imposed by our protocol. While the sample size in this study is limited, our results suggest that animals can effectively regulate their blood osmolality in a narrow range of stable values (300 to $320 \mathrm{mOsmo} /$ $\mathrm{KgH} 2 \mathrm{O}$ ) under these conditions. The strong relationship between water consumption and existing osmolality level further suggests that the animals may have achieved this stability by performing the task to obtain a sufficient level of water intake to reach the target osmolality range. This strongly implies that the water access control procedure we employed allowed the monkeys to maintain a stable hydration state.

Another remarkable feature of blood osmolality is that it indicates that hydration level increases as a function of water intake in a surprisingly linear manner even over the relatively rapid time course of an experimental session (Fig. 4). This is the first time the response of osmolality to water ingestion has been measured under the condition that mimicked fluid consumption during a standard reinforced behavioral task. The finding is consistent with a preexisting study in which monkeys drank ad libitum water after 24 hours water deprivation (Wood et al., 1980). In that case, plasma osmolality decreased very quickly about $20 \mathrm{mOsmo} / \mathrm{KgH} 2 \mathrm{O}$ (from 315 to 295) with $180 \mathrm{ml}$ water intake during 20 min. We found that the osmolality level decreased after delays of less than $30 \mathrm{~min}$ and that this decrease was quite linear, reaching about $8 \mathrm{mOsmo} / \mathrm{KgH} 2 \mathrm{O}$ on average, after a $150 \mathrm{ml}$ water intake. In some cases, however, we observed that the osmolality level did not decrease 
in a completely linear fashion as measured at this timescale. This might reflect fluctuations in the speed of water absorption from the gastro-intestinal tract. Of course measurement error ( \pm 1 mOsmo for our measurement technique) might also contribute to the nonlinearities we observed occasionally. While a delay between water intake and an observable decrease in osmolality level must exist, osmolality seems to provide a fairly precise reflection of the monkeys' hydration state on a timescale of minutes. Further study is necessary to understand how the changes in osmolality that occur as the result of water intake influence a subject's satiety and motivation to engage in the available reinforced task (Houpt et al., 1999).

On average, osmolality levels increased about 8 mOsmo from baseline levels during controlled water access. The data also suggest that the effect of controlled water access might vary in this regard from monkey to monkey (Fig. 3), although these differences were not statistically significant. Two possible explanations for these individual differences could be: 1) significant individual differences in the homeostatic process, and 2) a difference in the water restriction protocol used for monkey BT: We measured the minimal daily allocation in monkey BT at a slightly earlier period of behavioral training.

Our data revealed that: 1) The subjective values of fluid rewards are not likely to be stable within a short time period (less than $30 \mathrm{~min}$ ). The implicit assumption that this is the case which some papers appear to have made would be incorrect, and these data thus suggest that the effect of satiety for fluid reward should be included in data analyses if satiety could be a critical factor in an experiment. 2) The procedures widely used to control water intake do appear to be appropriate for maintaining the health of the subjects. Animals are able to regulate their fluid balance appropriately under these conditions. More extensive studies, however, would be necessary to establish a criterion level of blood osmolality for regulating controlled water access in laboratory primates. 3) It appears that the subjective values of fluid rewards, as modulated by the actual thirst of the animals, can be directly measured and manipulated. This measurement might thus allow for a better understanding of the neural basis of decision-making in the future. Further, we found that daily fluctuations of animal weight can also serve as a proxy for measurements of hydration state as is often hypothesized. The measurement of osmolality in addition to monitoring weight, however, could be an easy and efficient way to evaluate water access control when a precision osmometer is available and routine blood draws are possible.

\section{Acknowledgments}

The authors wish to express their gratitude to Samanta Shaw, Margaret Grantner and Lauren Grattan for technical assistance.

\section{References}

Armstrong LE. Hydration assessment techniques. Nutr Rev. 2005; 63:S40-S54. [PubMed: 16028571]

Bernardis LL, Bellinger LL. The lateral hypothalamic area revisited: ingestive behavior. Neurosci Biobehav Rev. 1996; 20:189-287. [PubMed: 8811717]

Berne, RM.; Levy, MN. Physiology. 3rd ed.. St. Louis: Mosby-Year Book, Inc.; 1993. p. 754-783.

Buggy J, Hoffman WE, Phillips MI, Fisher AE, Johnson AK. Osmosensitivity of rat third ventricle and interactions with angiotensin. Am J Physiol. 1979; 236:R75-R82. [PubMed: 434190]

Caraco T, Martindale S, Whitham TS. An empirical demonstration of risk-sensitive foraging preferences. Animal Behaviour. 1980; 28:820-830.

Critchley HD, Rolls ET. Hunger and satiety modify the responses of olfactory and visual neurons in the primate orbitofrontal cortex. J Neurophysiol. 1996; 75:1673-1686. [PubMed: 8727405] 
de Araujo IE, Kringelbach ML, Rolls ET, McGlone F. Human cortical responses to water in the mouth, and the effects of thirst. J Neurophysiol. 2003; 90:1865-1876. [PubMed: 12773496]

Dickinson A, Balleine B. Motivational control of goal-directed action. Animal Learning and Behavior. 1994; 22:1-18.

Evarts EV. A technique for recording activity of subcortical neurons in moving animals. Electroencephalogr Clin Neurophysiol. 1968; 24:83-86. [PubMed: 4169750]

Fuchs AF, Robinson DA. A method for measuring horizontal and vertical eye movement chronically in the monkey. J Appl Physiol. 1966; 21:1068-1070. [PubMed: 4958032]

Goldberg ME, Wurtz RH. Activity of superior colliculus in behaving monkey. I. Visual receptive fields of single neurons. J Neurophysiol. 1972; 35:542-559. [PubMed: 4624739]

Houpt TR, Yang-Preyer H, Geyer J, Norris ML. A rapid feedback signal is not always necessary for termination of a drinking bout. Am J Physiol. 1999; 276:R1156-R1163. [PubMed: 10198398]

Lau B, Glimcher PW. Value representations in the primate striatum during matching behavior. Neuron. 2008; 58:451-463. [PubMed: 18466754]

Mays LE, Sparks DL. Dissociation of visual and saccade-related responses in superior colliculus neurons. J Neurophysiol. 1980; 43:207-232. [PubMed: 6766178]

Minamimoto T, La Camera G, Richmond BJ. Measuring and modeling the interaction among reward size, delay to reward, and satiation level on motivation in monkeys. J Neurophysiol. 2009; 101:437-447. [PubMed: 18987119]

Platt ML, Glimcher PW. Neural correlates of decision variables in parietal cortex. Nature. 1999; 400:233-238. [PubMed: 10421364]

Platt ML, Glimcher PW. Responses of intraparietal neurons to saccadic targets and visual distractors. J Neurophysiol. 1997; 78:1574-1589. [PubMed: 9310444]

Rolls, BJ.; Rolls, ET. Thirst. Cambridge: Cambridge University Press; 1982.

Samejima K, Ueda Y, Doya K, Kimura M. Representation of action-specific reward values in the striatum. Science. 2005; 310:1337-1340. [PubMed: 16311337]

Simon SA, de Araujo IE, Gutierrez R, Nicolelis MA. The neural mechanisms of gustation: a distributed processing code. Nat Rev Neurosci. 2006; 7:890-901. [PubMed: 17053812]

Stedman, TL. Stedman's Medical Dictionary. 28th ed.. Philadelphia: Lippincott Williams \& Wilkins; 2006.

Thrasher TN, Keil LC. Regulation of drinking and vasopressin secretion: role of organum vasculosum laminae terminalis. Am J Physiol. 1987; 253:R108-R120. [PubMed: 3605376]

Wood RJ, Maddison S, Rolls ET, Rolls BJ, Gibbs J. Drinking in rhesus monkeys: role of presystemic and systemic factors in control of drinking. J Comp Physiol Psychol. 1980; 94:1135-1148. [PubMed: 7204702]

Wood RJ, Rolls ET, Rolls BJ. Physiological mechanisms for thirst in the nonhuman primate. Am J Physiol. 1982; 242:R423-R428. [PubMed: 7081465] 




Fig. 1. Increase of serum osmolality across a week

(A) Plots of serum osmolality across a week. Two series of samples for each of three monkeys are represented. (B) Plot of average change in serum osmolality from Monday baseline. (C) Plot of average serum osmolality across a week. * and **: $\mathrm{P}<0.05$ and $\mathrm{P}<$ 0.01 by two sample t-test against Monday level. Error bars indicate standard errors of the mean. (D) Plot of average daily water intake in the behavioral task and out of the task. (Note: The large Friday evening water supplement is not shown here.). The plots were normalized by the amount of minimal daily allocation of water (Mini) in each monkey DE and HU. 


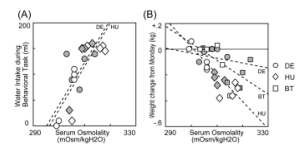

Fig. 2. Serum osmolality is correlated with both water intake during an eye movement task and weight change

(A) Plot of serum osmolality measured before performing eye movement task against total water intake during the task for monkeys DE and HU. (B) Plot of serum osmolality against weight change from Monday in three monkeys. Dashed line represents regression line in each monkey. 


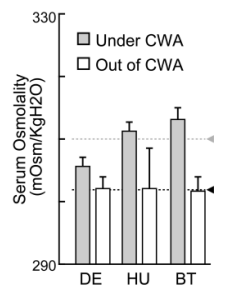

Fig. 3. Serum osmolality measured under and out of controlled water access

Average plot of serum osmolality in each monkey under and out of controlled water access (CWA). The data under CWA was same as in experiment 1. Error bars indicate standard errors of the mean. Gray and black triangles with dashed lines indicated average across monkeys under and out of controlled water access, respectively. 


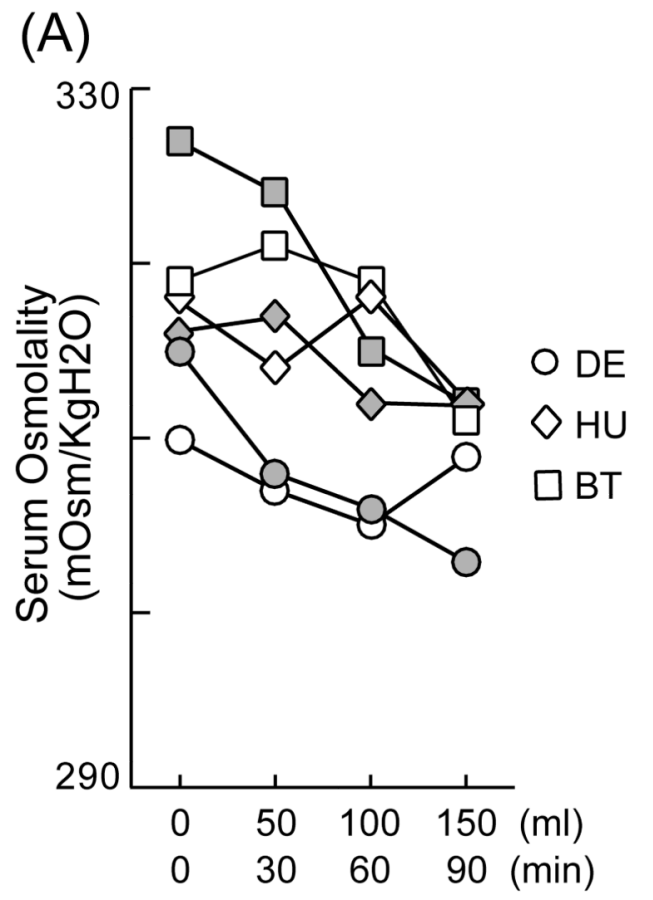

(B)

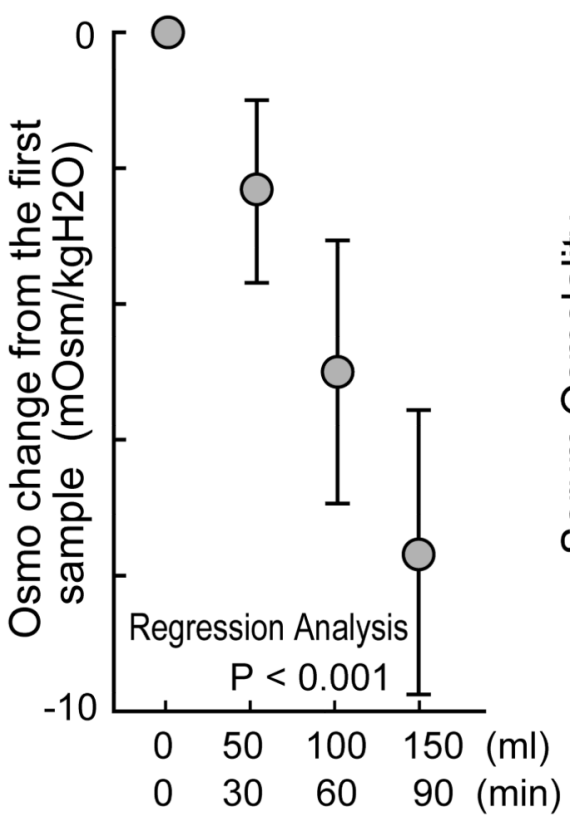

(C)

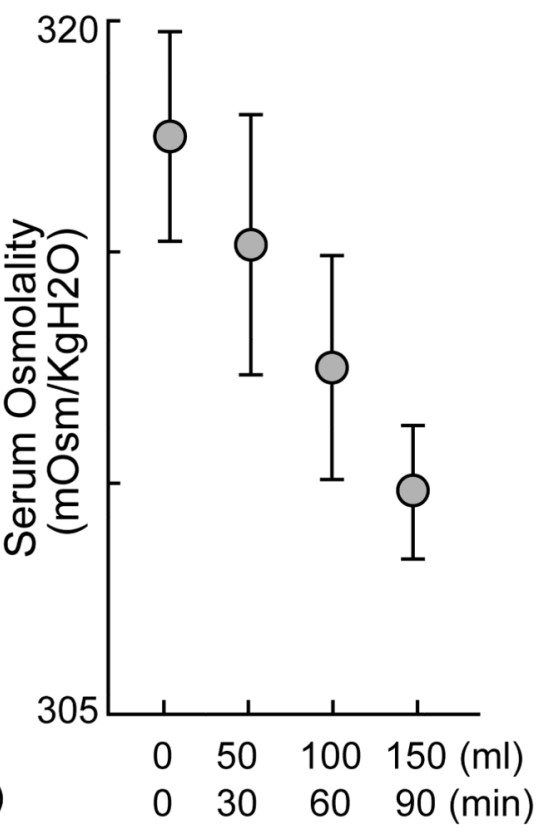

Fig. 4. Decrease of serum osmolality after water intake within a day (A) Plot of serum osmolality against water intake every 30min. The two series of samples for each of the three monkeys are represented. (B) Plot of average changes in serum osmolality from the first sample before water intake. (C) Plot of average serum osmolality again water intake every $30 \mathrm{~min}$. Error bars indicate standard errors of the mean. 